\title{
La Universidad Tardocolonial Latinoamericana: el caso de Santafé en la Nueva Granada, 1800-1810 ${ }^{1}$
}

\author{
Jorge Tomás Uribe Ángel ${ }^{2}$ \\ Universidad Pedagógica y Tecnológica de Colombia (Colombia) \\ Grupo de investigación ILAC \\ jorgeturibea@gmail.com
}

Recepción: 17/07/2013

Evaluación: 15/11/2013

Aceptación: 13/12/2013

Artículo de Revisión

DOI:http: / / dx.doi.org/ 10.9757/Rhela.22/06

\section{RESUMEN}

A fines del período colonial fue evidente que las iniciativas criollas en la educación superior reemplazaron a las provenientes de la metrópoli. Estas iniciativas antecedieron a las provenientes del grupo ilustrado en otros campos como la economía y especialmente en la política. Esos cambios, acaecidos antes de la Declaración de Independencia, pusieron de presente que los docentes neogranadinos, dentro de los cuales figuraban personajes que después ocuparon destacadas posiciones en el proceso de Independencia, ya no siguieron un programa determinado para el aprendizaje de los contenidos ilustrados al interior de las aulas. Al mismo tiempo, las autoridades españolas contaron con el criterio de los criollos para la adopción de planes de estudio, en especial para los relacionados con los centros de enseñanza de carácter provincial.

Palabras clave: Revista Historia de la Educación ilustración, educación superior, planes de estudio, criollos, independencia, periodo tardo colonial.

1 Este texto es resultado del proyecto de investigación Reformas y movimientos universitarios en Colombia SGI 1295, desarrollado por el grupo Ilustración en América Colonial - ILAC y financiado por la Dirección de Investigaciones de la Universidad Pedagógica y Tecnológica de Colombia.

2 Doctor en Ciencias de la Educación de la Universidad Pedagógica y Tecnológica de Colombia, RUDECOLOMBIA, Magíster en investigación interdisciplinaria en Ciencias Sociales de la Universidad Distrital, Abogado de la Universidad Javeriana. Líneas de investigación: Historia de la Educación. Miembro correspondiente de la Academia Colombiana de Historia, integrante del grupo de investigación Ilustración en América Colonial -ILAC, adscrito a la Universidad Pedagógica y Tecnológica de Colombia. 
The tardocolonial Latin America University: The case of Santafé in la Nueva Granada. 1800-1810
A universidade Tarda-coloniallatino-americana: O Caso de Santafé em Nova Granada. 1800-1810

\section{ABSTRACT}

At the end of the colonial period it was clear that the creole initiatives in the higher education replaced the metropolis ones. These initiatives preceded those of the illustrated group in other fields as the economy and especially in politics. These changes took place before the Declaration of Independence. These changes showed that neogranadinos teachers did not continue a program determined for the learning of enlighten contents into the classrooms. Then, these teachers had roles during the independence.

At the same time, the Spanish authorities were provided with the criterion of the Creoles for the adoption of study plans, especially, in relation with the centers of education of the towns.

Key words: Journal History of the Latin-American Education, enlightenment, higher education, tudy plans, Creoles, independence, slow colonial period.

\section{RESUMO}

Em fins do período colonial foi evidente que as iniciativas crioulas na educação superior substituíram as provenientes da metrópole. Estas iniciativas antecederam às provenientes do grupo ilustrado em outros campos como a economia e especialmente na política. Essas mudanças, ocorridas antes da Declaração da Independência, determinaram que os docentes neogranadinos, entre os quais figuravam pessoas que depois ocuparam destacadas posições no processo de Independência, já não seguissem um programa determinado para oensino dos conteúdos apresentados nas aulas. Ao mesmo tempo, as autoridades espanholas contaram como o critério dos crioulos para adoção de planos de estudos, em especial para os relacionados com os centros de educação de caráter provincial.

Palavras-chave: Revista História da Educação Latino-americana, ilustração, educação superior, planos de estudo, crioulos, independência, período tardo-colonial.

\section{INTRODUCCIÓN}

Con respecto a la educación superior en Santa fe, capital del virreinato de la Nueva Granada, por medio de una investigación titulada “La formación de la generación de Independencia a través de la Ilustración. 1774-1810", se ha llegado a la conclusión de que, dentro de la universidad, los saberes ilustrados no encontraron un campo propicio para su difusión de tal suerte que los criollos debieron aprehender esos saberes por fuera de las aulas. Si bien, en el caso de Santafé fueron las autoridades mismas, a través de la introducción de cátedras nuevas como la de matemáticas, o la introducción de planes de estudio las que propiciaron la enseñanza de los saberes ilustrados dentro de la universidad, razones de diversa índole dificultaron la consolidación de esos empeños. 
Celina Lértora corrobora lo ocurrido en Santafé. De acuerdo con ella, la inserción de los saberes ilustrad o en el mundo universitario colonial se efectuó principalmente por medio de dos vías, la modificación de los planes de estudio existentes, ${ }^{3}$ o la introducción de cátedras nuevas. ${ }^{4}$ En Santafé se intentaron, como lo veremos, no solamente ambas soluciones sino también una tercera, consistente en variar los contenidos de la cátedra de filosofía sin seguir ningún plan de estudios. Por lo demás, por medio del adaptacionismo y del eclecticismo, se implementaron nuevos métodos de enseñanza y textos modernos, y se diseñaron planes de estudio. Se trataba de traer "lo útil y práctico" al seno de la universidad, lo que abriría camino al pensamiento científico y a las corrientes sobre las cuales se estaba consolidando el estado moderno.

Además, si bien el aprendizaje de los saberes ilustrados se realizó fuera de las aulas, a fines del período colonial, poco antes del movimiento de Independencia, los saberes ilustrados, concretamente los que se impartían por medio de la cátedra de filosofía, ${ }^{5}$ la cual, por otra parte, constituía el fundamento de los estudios superiores, ya hacían parte de la enseñanza superior. Efectivamente, las directivas universitarias y los criollos se las habían arreglado para que esas materias, particularmente las que se relacionaban con la ciencia moderna, hicieran parte de los contenidos que se aprendían dentro de las aulas. Obviamente, no se trata de sostener que los saberes ilustrados habían penetrado de lleno dentro de los programas de estudio, sino que la evidencia misma como se presentaba a los ojos de la vanguardia intelectual de Santafé, hacía que la posición irreductible de los partidarios de la enseñanza tradicional, particularmente de la escolástica, comenzara a desmoronarse con carácter definitivo. ${ }^{6}$

En realidad, para llegar al punto en que los saberes ilustrados, en el último decenio de la dominación hispánica, penetraran en las aulas de los colegios mayores, y por iniciativa de los criollos, se había recorrido un largo camino que es necesario describir, a saber:

3 Ejemplo de modificación de los planes existentes es el ya citado propuesto por Francisco Antonio Moreno y Escandón en 1774.

4 Ejemplo de cátedra nueva fue la creación de la cátedra de matemáticas por José Celestino Mutis en 1762.Ver:J. C. Mutis,Discurso preliminar pronunciado en la apertura del curso de matemáticas, el día 13 de marzo de mil setecientos sesenta y dos, en Colegio Mayor del Rosario de Santa Fe de Bogotá(Bogotá:Ediciones Fondo Cultural Cafetero, 1982),35.

5 En la cátedra de filosofía, fundamental para seguir estudios en las facultades mayores -teología, derecho y medicina-, originalmente se seguía el plan basado en Aristóteles, en virtud del cual después de la lógica, se cursaba la metafísica y después la física. En el de Moreno y Escandón, la novedad consistía en la enseñanza de la física moderna, antes que la metafísica. Otra innovación importante con respecto al plan tradicional consistió en la introducción de la filosofía moral.

6 El último autor que sirvió de base para enseñar la escolástica en los Colegios Mayores de Santafé fue Antonio Goudin (16391695), teólogo y filósofo francés, dominico, de carácter tomista, cuya obra circuló profusamente en Francia y España. 
1. Desde la creación de la cátedra de matemáticas en el colegio del Rosario por José Celestino Mutis en 1762, hasta la redacción del plan provisional de estudios para los colegios mayores de Santafé, por Francisco Antonio Moreno y Escandón en 1774.

2. Desde la puesta en marcha del plan de Moreno y Escandón hasta el reemplazo de este por el plan de la Junta de estudios de 1779, de inspiración escolástica.

3. Desde la puesta en marcha del plan de 1779 , hasta la sustitución de la cátedra de derecho público, única innovación de carácter ilustrado que presentó este plan, por la derecho real en $1795 .^{7}$

4. Desde la creación de la cátedra de derecho real, hasta la aprobación del plan de estudios definitivo para la enseñanza de la medicina en el Colegio del Rosario en 1805. En este período, la aplicación del plan de estudios de 1779, con la creación de la cátedra de derecho real, empieza a desdibujarse. Se crean, además, otras cátedras como las de química ${ }^{8}$ y mineralogía, las de física y matemáticas en la escuela del mismo nombre, a cargo del catedrático Bernardo de Anillo, y las de dibujo y arquitectura, en la escuela del mismo nombre y a cargo también del catedrático Anillo.

5. La aprobación del plan definitivo de estudios para la facultad de medicina del Colegio Mayor del Rosario en 1805, ${ }^{9}$ redactado por José Celestino Mutis, primer plande estudios de carácter ilustrado que empezó a funcionar con carácter definitivo en los colegios mayores de Santafé.

6. En 1808 los rectores de los colegios mayores de Santafé informan que los colegios no se rigen por ningún plan de estudios. Asimismo, los catedráticos de filosofía reconocen que en la cátedra de filosofía los contenidos son de carácter ilustrado. Se presentó el abandono del texto de Goudin, de carácter escolástico, que de acuerdo con el plan de 1779 debía utilizarse en la cátedra de filosofía, y la adopción del texto de Christian Wolf, de carácter ilustrado, sustitución acaecida

7 Con la creación de la cátedra de derecho público se rompe la tradición iusnaturalista de carácter escolástico basada en Santo Tomás, y se introduce la del racionalismo que considera a Grocio como el creador del derecho natural.

8 La apertura de la cátedra de química fue una iniciativa presentada, con el apoyo de Mutis, por el rector del Colegio del Rosario, Fernando Caicedo y Rojas, y por Jorge Tadeo Lozano al virrey Mendinueta en 1801. Ver:Pedro María Ibáñez, Crónicas de Bogotá. Tomo II (Bogotá: Imprenta Nacional, 1915), 192.

9 Ver José Celestino Mutis, "Plan general de los estudios médicos, arreglado según las proporciones del país a la enseñanza de todas sus producciones subalternas, propuesto al Gobierno por don José Celestino Mutis, en su carácter de Regente de Estudios para el Colegio Mayor de Nuestra Señora del Rosario. Santafé, mayo de 1804".Real Jardín Botánico de Madrid, Archivo de la Real Expedición Botánica, Leg. 50-62/10, en Documentos para la Historia de la Educación en Colombia. Tomo VI. 1800-1806. Compilador Guillermo Hernández de Alba, (Bogotá: Editorial Kelly, 1985),178. 
alrededor de 1804.Los criollos asumen como propias las iniciativas de carácter ilustrado, y son consultados acerca de los planes de estudios que se pretende implementar en otras localidades del Nuevo Reino, como Medellín y Mompox.

Como se observa, hubo de recorrerse un largo camino, desde 1862 hasta fines del período colonial, al término del cual se hubo de reconocer la vigencia de aquellos contenidos quedefendía el movimiento ilustrado, y también la preponderancia que había adquirido el elemento criollo en las iniciativas de carácter educativo frente a la pasividad de las autoridades españolas en esa materia.

Justamente, el objetivo de este artículo es el de precisar de qué manera, a fines del período colonial, los criollos en el aspecto educativo, antes que en otras actividades, como la política y economía, habían tomado la iniciativa, de suerte que, cuando acaeció la revolución de Independencia, en el campo de la educación el estamento criollo había asumido la iniciativa en cuanto a la naturaleza de los saberes que se dictaban en las aulas. Esta postura determinó que ya desde ese entonces, los estudiantes de los Colegios Mayores no tuvieron que aprender los contenidos ilustrados fuera de las aulas sino dentro de ellas.

Para la realización de este artículo se ha seguido el método histórico por medio de la utilización de fuentes de archivo impresas.

\section{Iniciativas criollas}

Durante los últimos años del período colonial, y especialmente luego de la aprobación del plan de estudios de Medicina en 1805, se hizo evidente que, en materia educativa, los criollos, sin desobedecer los dictados de la Corona, habían tomado la iniciativa. ${ }^{10}$ Esta actividad se desplegó, especialmente, en la redacción de varios planes para el desarrollo de diversos saberes, especialmente la minería; ${ }^{11}$ la asesoría prestada con relación a planes de estudio que se redactaron en provincia y, sobretodo, el abandono del plan

10 Generalmente, la historiografía destaca la iniciativa que tuvieron los criollos en la economía, y especialmente en la política. La iniciativa de los criollos en materia educativa ha recibido poca atención.

11 Ver: sobre la minería, entre otros, Juan José Delhuyar, "Natural, breve, nuevo y muy seguro método de beneficiar la Plata por Azogue. Comunicado por (Don) Jayme Navarro desde la Montuosa Baja", enCatálogo del Fondo Documental José Celestino Mutis del Real Jardín Botánico. Instituto de Cultura Hispánica. (Madrid: Registro 3466, 1995), 308. 
de estudios de 1779. La iniciativa desarrollada por los criollos en materia educativa es la última de las etapas que durante la Colonia presentó la enseñanza de los contenidos ilustrados dentro de las aulas.

Ya desde 1804, en los Colegios Mayores, se había abandonado el texto de Goudin, de corte escolástico, y se había acogido el de Wolf. ${ }^{12} E$ ste viraje significó que, por lo menos en aspectos relacionados con la enseñanza de las ciencias, la postura tradicional resultaría insostenible.

Un reconocimiento explícito de que en los colegios mayores no se seguía ningún plan de estudios se efectuó en 1808 en un informe presentado por los rectores de ambos colegios de Santafé, en respuesta a una solicitud del virrey. Así, Nicolás Cuervo, de San Bartolomé, el 27 de julio de ese año afirmó que "sin embargo de no haberse adoptado hasta aquí finalmente algún plan de estudios y por ello no podrá este Rector complacer a V. E., remitiendo a su superioridad la copia que se pide" También el rector del Rosario, Vicente de la Rocha, en comunicación del 12 de agosto, informó que "en esta capital no ha habido un plan que rija los estudios".

Como se les preguntó si seguían el plan ilustrado que había elaborado en 1787 el arzobispo virrey Antonio Caballero y Góngora, ambos rectores informaron que como éste se había redactado para servir de apoyo a la universidad pública, y tal circunstancia no se había presentado, no le habían dado curso al plan mencionado.

Lo anterior nos lleva a preguntarnos por la vigencia que había tenido el plan de la Junta de Estudios de 1779. Es evidente que, de acuerdo con la documentación allegada sobre el particular, y teniendo en cuenta las protestas formuladas por algunos docentes acerca del contenido y los textos empleados en la cátedra de filosofía, y los trámites realizados para la puesta en marcha de la cátedra de derecho público, por lo menos hasta la década de 1790, el plan de 1779 conservaba su vigencia, vigencia que perdió en el curso de la primera década del siglo XIX. Por ello, ambos rectores no habían vacilado en informar que en 1808 los estudios no se regían por plan alguno, con sus naturales consecuencias, en particular en relación con la cátedra de filosofía.

12 Christian Wolff (1679-1754) fue un filósofo alemán que influyó en las teorías de Kant. 
En cuanto hace a la enseñanza de la filosofía, en el Colegio de San Bartolomé, ${ }^{13}$ el rector comunicó que el método que se seguía era el

dispuesto por el actual catedrático D. D: Custodio García Rovira, a quien a confiado formación por ser sujeto experimentado acerca de los progresos, e pueden resultar al público de la elección de materias filosóficas por haber ese catedrático leído dos cursos y con el que van a empezar tres; habiendo los cursantes dado pruebas muy exactas de su su aprovechamiento y esperanzas bien fundadas de mejorar la ilustración del Reino.

En relación con el Colegio del Rosario, también la iniciativa en materia de la cátedra se manejaba autónomamente.

[La filosofía ${ }^{14} q u e$ en unos días ha hecho tantos progresos en Europa, no ige al parecer otra elección que la de buen catedrático, porque la razón sola de lo que nuevamente se descubre pide que se le deje a él la elección de autores para mejor instrucción de los jóvenes, lo que tiene que lograr principalmente en la física".

En el caso de San Bartolomé, anexo al informe del rector, fue el propio Custodio García Rovira quien explicó cómo él, en su calidad de catedrático, estaba llevando a cabo una serie de innovaciones en la enseñanza, particularmente en la de la física. “En el primer año enseñaré la Lógica, Metafísica y Moral; en el segundo, la Aritmética, Álgebra, Geometría y Trigonibetría; en el tercero, Física universal, particular y los Elementos de Química hasta donde alcance". Los textos sugeridos eran, para la aritmética y la geografía, el de Blon y Bayls, ${ }^{15}$ para las otras asignaturas el de Altieni y el Curso de León. Además, los de Paulian, ${ }^{16}$ Pará de Fanjas ${ }^{17}$ y Brisson. ${ }^{18}$ Otros autores eran Wolffio y Lavoisier. ${ }^{19}$ Con respecto al uso en la cátedra de ciertos autores, anotó García Rovira:

13 Cuervo y García, “Informe sobre el plan...,156.

14 De la Rocha, "Informe sobre el plan..., 161.

15 Benito Bails, Arquitecto y matemático español de la Ilustración, (1730-1797).

16 PAULIAN Aimé-Henri. (1722-1801) Autor de Système general de philosophie extrait des ouvrages de Descartes et de Newton 1769 y de un Dictionaire de Physique. Se encuentra en la BibliotecaNacional.

17 Francois du Phanjas, Teoría entiumin sensibilium sive methaphysica universa sacra et prophana,Fondo Antiguo, Biblioteca Nacional.

18 Jacques Brisson ,Diccionario Universal de Física, 1801, Fondo Samper, Biblioteca Nacional en francés Dictionaire raisoné de physique, 1781, Fondo Antiguo Biblioteca Nacional.

19 Antoine Laurent de Lavoissier (1743-1794) Biólogo y economista francés, considerado el creador de la química moderna. 
Es cierto que estos conocimientos se les darían mejor siguiendo al Condillac $^{20}$ o Foronda en las matemáticas a Lacroix,, en la Metafísica y Moral al Pará, en la Física al Brisson, o al mismo Pará.Pero atendidas las circunstancias en que nos hallamos, es impracticable este proyecto por lo que estos autores se hallan generalmente, unos en francés y otros en castellano, lo que es ciertamente incompatible con nuestros estudios en latín.

Por otra parte, por circunstancias de tiempo, afirmó García Rovira que no se podían dictar clases de Botánica, Mineralogía e Historia Natural, asignaturas que se deberían abordar, según el catedrático, fuera del ciclo regular.

Con respecto a la modalidad de solicitar el parecer de los criollos, con respecto a los planes de estudio que se redactaban en provincia, la primera intervención se refirió a los comentarios con respecto al plan de estudios presentado para el nuevo Colegio Anexo a la Fundación de San Francisco de la villa de la Candelaria de Medellín. El claustro de doctores de la Universidad Tomística solicitó a Camilo Torres ${ }^{22}$ un análisis del mencionado plan. Este se manifestó sobre el particular con un estudio realista sobre el asunto, en el cual ponía de presente las dificultades resultantes de poner en la práctica un plan demasiado ambicioso que no se compadecía con los recursos que, por ejemplo, en términos de catedráticos y textos, podía ofrecer la villa. También un joven criollo ilustrado, José Manuel Restrepo, ${ }^{23}$ en nombre del cabildo de la villa de Medellín, manifestó la opinión que a los regidores les mereció el plan de estudios mencionado.

Esta función de conceptuar acerca de las novedades que en materia educativa fueran surgiendo, en especial en relación con los estudios de educación superior, supuso, en primer lugar, que a los dirigentes educativos y catedráticos criollos se les reconocían méritos para opinar sobre materias tan delicadas como los planes de estudios de las instituciones de educación

\footnotetext{
20 Etienne Bonnot de Condillac, Economista y filósofo francés. Actuó durante la época de Napoleòn (1715-1780).

21 Silvestre Francois Lacroix, Traité élémentaire du calcul des probabilités, Fondo Samper, Biblioteca Nacional.

22 Camilo Torres Tenorio estudió en el Colegio Mayor de Nuestra Señora del RosarioDerecho y fue catedrático de la misma institución. Uno de los protagonistas de la revolución del 20 de julio de 1810 escribió el Memorial de Agravios. Durante la Primera República, dirigió el bando federalista y fue presidente de las Provincias Unidas de la Nueva Granada, ejecutado durante el régimen del terror.

23 José Manuel Restrepo estudió en el Colegio de San Bartolomé, donde se graduó de abogado. Naturalista, colaboró en el gobierno de Antioquia durante la Primera República. Fue gobernador de Antioquia, nombrado por Bolívar Secretario del Interior y Relaciones Exteriores. Se distinguió como historiador por haber escrito, entre otros,Historia de la Revolución de la República de Colombia en la América Meridional(1858).
} 
superior, antecedente que recordaba las diligencias que llevaron en primer lugar a la aprobación del plan de Moreno y Escandón, y, posteriormente, la intervención de la llamada Junta de Estudios de 1779, cuando se dispuso el cambio del Plan de estudios de Moreno. Se avalaba la capacidad de los jóvenes criollos para hacerse cargo de la educación superior, en respuesta al clamor de Francisco Antonio Zea, ${ }^{24}$ formulado quince años antes, a favor de una reforma en la educación y sobretodo en relación-en el caso del Colegio Universidad de Mompox-, con la cátedra de filosofía, núcleo académico de las instituciones de esa categoría. Así, se fueron recogiendo los aires de renovación que a ese respecto empezaban a penetrar definitivamente en los colegios mayores santafereños.Por otra parte, esta innovación subraya la tendencia de tipo centralista de someter a las autoridades de Santafé a los asuntos relativos a la educación superior. Es importante precisar también que la única universidad que funcionaba en el Nuevo Reino era la Tomística.

Con respecto a la cátedra de filosofía, la modalidad de consulta se presentó cuando el plan de estudios en esa asignatura, presentado por el Colegio Universidad de Mompox fue sometido a la consideración del claustro menor de la Universidad Tomística. Este, a su vez lo pasó a los catedráticos de filosofía de la Universidad y Colegios Mayores de Santafé, Custodio García Rovira, del Colegio Mayor de San Bartolomé, Ramón Bustamante, del Colegio del Rosario, Fray José de Jesús Saavedra, vicerrector de la Universidad (8 de octubre de 1806). ${ }^{25}$ Asimismo, opinaron en su momento José Domingo Duquesne ${ }^{26}$,rector de San Bartolomé (9 de mayo de 1807), Vicente de la Rocha, rector del Rosario (1 de febrero 20 de junio de 1808), ${ }^{27}$ Nicolás Cuervo, rector de San Bartolomé (17 de marzo de 1808). ${ }^{28}$

A pesar de que el plan no se aplicó en Santafé, es importante conocerlo, puesto que fue sometido al criterio de pedagogos que habitaban en la ciudad. Básicamente, el plan, firmado por Cándido Nicolás Girón, procurador

24 Francisco Antonio Zea (Medellín, 1766 - Bath, 1822). Estudió en Popayán y en el Colegio Mayor de Nuestra Señora del Rosario. Miembro de la Expedición Botánica. A raíz de la conspiración de Nariño (1794) emigró a España. Luego de la Revolución de Independencia, se unió a Bolívar y participó en el Congreso de Angostura. En 1820 fue enviado a Europa, pero fue desautorizado por haber facilitado la contratación de empréstitos que fueron gravosos para la Gran Colombia.

25 Custodio García, Ramón Bustamante y Fray José de Jesús Saavedra, "Conceptos de los catedráticos doctor Custodio García Rovira, del Colegio Mayor de San Bartolomé, doctor Ramón Bustamante, del Colegio Mayor del Rosario y Fray José de Jesús Saavedra, vicerrector de la Universidad", en Documentos para la historia de la educación en Colombia compilado por Guillermo Hernández de Alba. 103-117. (1985)

26 José Domingo Duquesne, (Bogotá 1745-Bogotá 1822). Estudió en el Colegio Mayor de San Bartolomé, donde se doctoró en Teología y Derecho Canónico. Escribió una Disertación sobre el calendario de los muiskas y una gramática chibcha. Fue detenido durante el régimen del terror. Se le considera el fundador de la antropología en Colombia.

27 De ka Rocha, "Informe sobre el plan",133-151.

28 Cuervo y García. “Informe sobre el plan “151-155. 
del número, ${ }^{29}$ que se cursaba para la cátedra de filosofía, en tres años, contemplaba los siguientes aspectos: en el primer año se estudiaría la lógica en dos meses, "de un modo enunciativo, sin meterse en la menor cuestión o disputa", de acuerdo con el abate Andrés Corsini, ${ }^{30} \mathrm{Jacquier}^{31}$ y el Genuense, "por otro nombre Antonio Genovesi". Los nueve restantes se dedicaban a la Aritmética, la Geometría, la Trigonometría, el Álgebra, Secciones Cónicas y Cálculo Infinitesimal. También, en ese primer año, en la que en el plan se denomina Instrucción Secundaria, se repasaba el catecismo de Fleury, la historia de España por el compendio del padre Isla, "la Conquista de América compendiada en inglés y traducida con elogio al castellano". Finalmente, en el primer año también se realizaba una "instrucción epistolar o de escritorio", y una de dibujo. En el segundo año se abordaba el conocimiento de la física general y también de la física particular. En la instrucción secundaria se aprendían rudimentos de historia natural, de acuerdo con Bosnet; economía y política; geografía, instrucción epistolar, historia "de acuerdo con Mariana ${ }^{32}$ y Saavedra", dibujo, y, en la lección de refectorio, los "tomos de Pluche". En el tercer año se aprendían los tratados del fuego, aire y agua, geografía, botánica, animástica, incluyendo aspectos relacionados con los animales y el hombre, y finalmente ética. La instrucción secundariacubría elementos de química e historia natural de acuerdo con la obra de Fourcroy, ${ }^{33}$ la de los establecimientos ultramarinos de Almodóvar, nociones de meteorología, por medio de la formación de diarios y del uso de instrumentos como el termómetro, el barómetro y el ventivol o velera de rumbos, y también por medio de los calendarios hortenses. Durante meses se ejercitaban en la elaboración de "discursos filosóficos, sobre cualquier objeto de historia natural";además se ejercitaban para la práctica del tratado de ética y para la formación "de los planes domésticos o de régimen privado", de acuerdo con la ocupación:"eclesiástico, hacendado, comerciante, militar etc o u simple particular". Se seguía con el dibujo, y en la lección de refectorio se utilizaba la historia natural de Buffon, ${ }^{34}$ en la parte de los animales.

\footnotetext{
29 Actuó como testigo en Santafé en las informaciones de varios aspirantes a ingresar como colegiales en San Bartolomé. Para la época en que elaboró el plan contaba entre cuarenta y cincuenta años. Ver Real Colegio Mayor y Seminario.

30 Andrés Corsini (1302-1374)fue un carmelita italiano de la orden de los carmelitas. Fue santificado.

31 FrancoisJacquier (1711-1788) matemático francés de la Orden de Mínimos, escribió comentarios a la obra de Isaac Newton.

32 Juan de Mariana S.J. (1536-1624),historiador, escribió Historia General de España(1601).

33 Antoine-Francois de Fourcroy (1755-1809) fue un químico francés. Ministro de Instrucción Pública, participó en la creación de la Escuela de Medicina y la Escuela Politécnica.

34 Georges Louis Buffon, (1797- 1788), fue un botánico, matemático, cosmólogo francés, que estableció un sistema de clasificación de las plantas posterior al establecido por Linneo. Escribió Historia natural, general y particular.
} 
El ambicioso plan pretendía que el estudiante efectuara un rápido recorrido por las diferentes ramas del saber, sin profundizar en ninguna, y buscaba, además, brindarle una formación de carácter integral que le permitiera desempeñarse adecuadamente en las distintas instancias de la vida, tanto en el orden profesional como en el orden privado y por medio de un contacto frecuente con la naturaleza. Pese a la carencia de tiempo, que imposibilitaba al aprendiz ahondar en los diferentes saberes, el plan ofrecía una perspectiva de aprendizaje bastante amplia, fomentando la aproximación a lo útil y lo práctico ytambién ejercitando al estudiante en la observación y en la experimentación.

- De esa suerte, se empezaba a abandonar la estructura que hasta ese momento había inspirado la formación superior desde la Edad Media: el énfasis en escribir bien, hablar bien y pensar bien. Lo que importaba, de acuerdo con el plan, era la preparación de una juventud que pudiera afrontar con solvencia y por medio de una información sumaria, los aspectos prácticos de la vida, por medio de la insistencia en diferentes destrezas que no habían formado parte hasta ese momento de la formación universitaria, ni siquiera de los planes ilustrados que se habían puesto en funcionamiento en Santafé, en los cuales, con respecto a la cátedra de filosofía, la innovación central se había dirigido a la enseñanza de la física. En el plan de Mompox seguía siendo importante el ejercicio de la facultad de pensar bien; pero al lado de ella se aprendía cómo escribir una carta, cómo llevar cuentas, cómo levantar un plano, cómo distinguir una especie vegetal, cómo llevar un registro meteorológico, cómo desempeñarse adecuadamente en el entorno doméstico, etc. ¡Cuán diferente era el horizonte en el cual hasta ese momento se había enmarcado el proceso de enseñanza con su insistencia en el cultivo del latín, en el abuso del silogismo, en el énfasis en la ostentación de los actos académicos, en el culto de la memoria y en la reverencia hacia lo puramente libresco! Ese plan, en cambio, irrealizable evidentemente en muchos aspectos, dada la falta de tiempo, y por carencia de catedráticos capacitados, constituía, sin embargo, un estímulo para el desarrollo de la imaginación por medio de las ciencias útiles y hasta cierto punto también, una incitación a abandonar la estrecha opción para la elección de profesión entre el sacerdocio, la jurisprudencia y la 
medicina. Un científico o un comerciante también encajaban dentro del espectro que señalaba el proyecto.

También es importante detenernos en las opiniones de los directivos y catedráticos mencionados, por cuanto nos ilustran sobre varias particularidades acerca del estado en que se encontraban los estudios de filosofía a fines del período colonial. Las opiniones reflejan varias tendencias, nacidas de la condición, eclesiástica o seglar, de quienes expresan la opinión, la edad, etc. Así, por ejemplo, mientras la mayoría de las opiniones concuerdan en que el clima de Mompox constituía un factor limitante, para el rector del Rosario, el clima cálido había sido un estimulante para el desarrollo de la civilización árabe. Para De la Rocha, desde el punto de vista de la disposición horaria, el plan resultaba irrealizable. Más bien, ${ }^{35}$ el examen del plan constituyó para el rector del Rosario un pretexto para lamentarse acerca de la escasa dedicación de los estudiantes, dado lo prolongado de las vacaciones, y de los asuetos, anotando que el padre Isla, "excitado de una justa curiosidad por saber el tiempo que se desperdiciaba, tuvo la prolijidad un año de ir apuntando los días de clase y al fin de él solo hallo en lista noventa y un días, esto es tres meses". Por lo demás, los estudiantes solían emplear la mayor parte de ese tiempo en la preparación de los actos de conclusión, descuidando el resto de sus deberes académicos. Otro aspecto que recuerda De la Rocha es la dedicación que en su momento, durante la aplicación del plan de Moreno y Escandón, había empleado Eloy Valenzuela en la cátedra de filosofía que dictó en el Colegio del Rosario durante la aplicación del plan de Moreno y Escandón, antecedente que constituía un buen presagio para el experimento que se intentaba adelantar en Mompox. Termina De la Rocha asegurando que, así como al sistema aristotélico le había llegado su hora, lo propio había ocurrido con el de Descartes, y que a la "atracción Newtoniana también le llegará su vez, aunque tarde", ${ }^{36}$ Por lo demás, el rector advierte que la decadencia experimentada últimamente por la "literatura" se debe más bien a la división de partidos que "a la falta de peripato". Pues "desterrado Aristóteles de Europa, era preciso que se proporcionase un asilo, y ninguno más seguro que la América", ${ }^{37}$ pues, al fin y al cabo, "este método no es reprobable por lo que hace a la lógica, metafísica, ética y matemática pura, pero no se puede adaptar en la física" ${ }^{38}$ señalándose que no hay "un solo moderno que haya deser-

\footnotetext{
35 De la Rocha, “Informe sobre el plan..., 134.

36 Ibid, 141.

37 Ibid.

38 Ibid, 142.
} 
tado de su escuela, cambiando las demostraciones geométricas por formas y unidades, el estudio de la naturaleza sensible por el de la materia primera y formas sustanciales" ${ }^{39}$

Sin lugar a dudas, de los literatos consultados, el que manifiesta un entusiasmo sin reservas por el plan es Custodio García Rovira, ${ }^{40}$ para quien "si este plan se considera tanto en sus materias, como en la escuela, con que previene se deben enseñar, no se puede escoger mejor para un colegio". A pesar de que se siente tentado a afirmar que le enseñanza de las matemáticas debería preceder a la de la lógica, acaba reconociendo que "ninguna ciencia o arte se puede poseer sólidamente siniestra, rectificados los pensamientos del hombre y esto no se puede conseguir sin la verdadera lógica, que es el arte de pensar bien". ${ }^{41}$ Sin embargo, García Rovira expresa que el plan, por su prolijidad, no está al acceso del estudiante medio, lo cual se podría evitar mandando "que se omitan aquellas partes, que se pueden aprender con más facilidad fuera del aula y del Colegio". ${ }^{42}$

1. El concepto del lector y vicerrector de la Universidad de Santo Tomás, Fray José de Jesús Saavedra, reconoce las bondades del plan, pero lamenta la falta de la metafísica y sugiere incorporar "otros principios de ética más fuerza de los propuestos como son por ejemplo sobre la libertad del alma, sobre la necesidad de una religión determinada". En el mismo sentido, se pronuncia el rector del Colegio de San Bartolomé, Domingo Duquesne, en mayo de 1807, al indicar que el plan "le ha cerrado la puerta a la metafísica [...]No se dice de Dios una sola palabra,[...] tampoco se dice nada del alma racional, [...] el hombre debe al autor del plan muy pocas palabras", aparte de manifestar que el proyecto es inconducente para seguir los estudios en las facultades mayores, concretamente jurisprudencia y teología. Duquesne, que en 1791había escrito un texto denominado Historia de un Congreso Filosófico tenido en Parnaso por lo tocante al Imperio de Aristóteles, donde se manifestaba acerbo enemigo del peripato, y donde proponía un nuevo método para la enseñanza

\footnotetext{
39 Ibid, 143.

40 Custodio García Rovira (1780-1816) Natural de Bucaramanga. Estudió en el Colegio Mayor de San Bartolomé y fue profesor de matemáticas, filosofía, metafísica y moral. En 1812 fue elegido gobernador del Socorro. Presidente de las Provincias Unidas de la Nueva Granada, fue sacrificado durante el régimen del terror.

41 Ibid., 105.

42 Ibid., 107.
} 
de la filosofía, cuando expone su concepto en esta oportunidad en relación con esas mismas materias, se muestra más conservador que el resto de los "literatos". Su concepto es, con mucho, el más extenso de todos; no vacila en hacer una crítica pormenorizada de carácter exegético de cada uno de los puntos que le merecen reparos. Se queja de que la lógica solamente se enseñe de manera enunciativa, de que en los estudios de ética falte la dogmática, de que en los tratados de animástica los aspectos relativos al alma racional no se traten a fondo. En relación con el estudio de la economía opina que si se trata "de la economía y política pertenecientea la denominación y gobierno de los estados en ninguna manera debe permitirse y apenas podría proponerse una materia más peligrosa".

Le parecen improcedentes la instrucción epistolar, propia para la formación de "papelistas y tinterillos", la de traer a colación anécdotas para introducir la enseñanza de la historia, la de los planes domésticos, (sugiere que si se trata de un eclesiástico lo procedente es acudir a un ayo). En fin, Duquesne conceptúa que el plan es adecuado "para crear jóvenes útiles a la sociedad, en el comercio, minas, agricultura, en una palabra en todos los ramos de la industria" ${ }^{43}$ pero no es procedente si se trata de formar estudiantes que quieran pasar a las facultades mayores.

Del examen de los conceptos formulados por los directivos universitarios y catedráticos que fueron convocados para analizar el plan de estudio de la cátedra de filosofía del colegio de Mompox se concluye que existe unanimidad acerca de la necesidad de modificar los estudios de física-el aspecto más novedoso de los planes ilustrados y con respecto al cual desde la escolástica se habían erigido críticas durante los últimos treinta años-, la cual debe enseñarse en el claustro. Es importante la opinión formulada por Duquesne en la medida en que se trata de un plan donde se privilegia lo útil y lo práctico, que se pone al servicio, no de la formación de las profesiones tradicionales, -ligadas, como se sabe, a perpetuar el statu quo por medio de la reiteración de saberes tradicionales que son a su vez manejados por una élite también tradicional sino de perfeccionar una formación por medio de un conjunto de saberes de orden práctico secularmente alejados de las aulas universitarias.

43 Ibid., 131. 
Sin embargo, se debe precisar que, no obstante la novedad en los contenidos cuya enseñanza se ofrece en el plan, todavía quedaba un rezago de la vieja estructura aristotélica de los saberes. La prioridad concedida a la lógica, como saber por medio del cual se debían iniciar los estudios; la nostalgia que despierta en algunos comentaristas la ausencia de la metafísica; el espacio concedido a la animástica; ${ }^{44}$ el hecho de que la serie de conocimientos por medio de los cuales se trataba justamente de reemplazar la física aristotélica, siguieran recibiendo el apelativo de física; constituyen todos reminiscencias de esa estructura aristotélica del conocimiento al que hemos aludido y que toca justamente en el meollo de la escolástica, el mal que se trataba de remediar. Seríamos, sin embargo, injustos sino admitiéramos que el plan constituyó un avance notorio, en la medida particularmente en que abrió un espacio a la enseñanza de lo útil y lo práctico, una opción que no se había contemplado seriamente como parte del proceso del aprendizaje impartido en los colegios santafereños. En estos, el ideal de enseñanza apuntaba todavía, con carácter hegemónico, hacia la formación de profesionales en aquellas disciplinas tradicionales que reforzaban el statu quo: el sacerdocio, la jurisprudencia y la medicina.

La participación creciente de los criollos en las iniciativas de carácter educativo, motivada, en parte, como lo acabamos de ver, por las consultas que se les formularon con ocasión de los planes de estudio presentados respecto de instituciones educativas de carácter regional, tanto en Medellín como en Mompox, nos muestran cómo la Corona cada vez tenía menor injerencia en las cuestiones educativas. Si bien, las autoridades seguían teniendo la última palabra en este aspecto, los criollos, inclusive cuando faltaban disposiciones sobre una determinada materia, se las ingeniaban para actuar por cuenta propia. Ello es evidente, por ejemplo, en el caso de los planes de estudio. Ya a fines del período colonial, el plan aprobado en 1779, de carácter escolástico, no tenía vigencia. Los criollos sustituyeron el texto de Goudin por el de Wolf, sin que mediara ninguna disposición al respecto. A pesar de que la aprobación del plan para la facultad de Medicina del Colegio del Rosario a principios de la década de 1800 había suministrado una base jurídica para modificar la cátedra de filosofía, los directivos de los colegios no habían tenido en cuenta esos cambios cuando afirmaban que se carecía de un plan de estudios.

44 La inclinación hacia la animástica nos recuerda que, con la Ilustración, especialmente en la Nueva Granada, se privilegiaron las ciencias naturales. 
Finalmente, vale la pena resaltar que, así como en Santafé se fue formando una red de abogados con amplia influencia en el resto del Nuevo Reino, también la ciudad, que en su condición de capital del virreinato era también el centro de la vida educativa, sirvió también para agrupar un grupo de criollos ilustrados versados en cuestiones educativas que posteriormente adquirirían notoriedad en el proceso de independencia tales como Francisco José de Caldas, ${ }^{45}$ Camilo Torres, Custodio García Rovira, José Manuel Restrepo. Ellos, oriundos de provincia, irradiaban, sin embargo, sus luces desde Santafé.

Definitivamente, a fines del período colonial, los saberes ilustrados habían penetrado al interior de las aulas, donde, sin embargo, en varios aspectos todavía campeaba la enseñanza de carácter tradicional.

\section{CONCLUSIÓN}

A fines del período colonial, los criollos habían asumido la iniciativa en el campo de la educación, especialmente en la redacción del plan de estudios de medicina de 1805, la redacción de varios planes para el desarrollo de nuevos saberes, especialmente la minería, la asesoría prestada con relación a planes de estudio y, sobre todo, el abandono del plan de estudios de 1779.

Sobre la inexistencia de planes de estudio estaban acordes los rectores de ambos colegios en Santafé.

En la iniciativa que en materia educativa asumieron los criollos, debe destacarse la participación de figuras que luego asumirían una importante participación política en la Independencia, tales como Camilo Torres Tenorio, Custodio García Rovira, José Manuel Restrepo.

1. Se adoptó la modalidad de solicitar a los criollos su opinión en relación con los planes de estudio que se redactaban en provincia, entre otros los de la villa de la Candelaria de Medellín y la villa de Santacruz

45 Francisco José de Caldas (Popayán 1768-Bogotá 1816), estudió en el Colegio Seminario Mayor de Popayán y en el Colegio Mayor de Nuestra Señora del Rosario. En 1801 entró en contacto con Mutis, de quien se considera el discípulo más aventajado. Conoció a Humboldt pero no consiguió que este lo llevar a Europa. Colaboró en la Expedición Botánica y recorrió el sur de Colombia y el Ecuador. Fundó y dirigió el Semanario de la Nueva Granada, primer periódico científico de Colombia. Víctima del régimen del terror, fue ejecutado. Conocido como el sabio Caldas, es el símbolo de la ciencia en nuestro país. 
de Mompox. En relación con este último, el plan encauzaba a los alumnos hacia el aprendizaje de lo útil y lo práctico, en detrimento de los saberes tradicionales, que restringían la elección de carreras en disciplinas como el Derecho, la Medicina y las Órdenes Sagradas.

No obstante el carácter innovador de los planes, todavía quedaron reminiscencias de la estructura aristotélica de la enseñanza.

\section{REFERENCIAS}

Cuervo, Nicolás. “El canónigo doctor Nicolás Cuervo, rector del Colegio de San Bartolomé, opina sobre las constituciones y el Plan de Estudios del Colegio de Mompós" en Hernández de Alba Guillermo Documentos para la Historia de la Educación en Colombia.1985

Cuervo, Nicolás y Custodio García Rovira, "Informe sobre el plan de estudios seguido en Colegio de San Bartolomé. 1808", en Hernández de Alba Documentos para la Historia de la Educación en Colombia. Volumen VI 1985.

De Restrepo, José Félix "Para el ingreso a los estudios de filosofía" En Papel Periódico T. I. No. 45 (1791)

Lertora, Celina. "Notas sobre la filosofía académica preilustrada en Nueva Granada", en Archivos y documentos para la historia de la educación colombiana compilado por Olmedo Vargas. Tunja: Rudecolombia, 2000.

Pacheco, Juan Manuel S. J. Sobre la Ilustración. Caracas: Universidad Católica Andrés Bello.

Quintero Esquivel, Jorge Eliécer P. “La huella de Christian Wolf en la Educación Neogranadina" En Revista Historia de la Educación Colombiana No. 2 (1999)

Quevedo Vélez, Emilio. "José Celestino Mutis y la medicina". En Mutis y la Real Expedición Botánica del Nuevo Reyno de Granada. Real Jardín Botánico. Madrid: Consejo Superior de Investigaciones Científicas, 1992.

Quevedo Vélez, Emilio. “La medicina en el Nuevo Reino de Granada”. En Historia Social de la Ciencia en Colombia. Volumen VII. Bogotá: Colciencias, 1993.

Restrepo, José Manuel. “El doctor José Manuel Restrepo, en nombre del cabildo de la villa de Medell\{in, manifiesta la opinión que a los regidores ha merecido el Plan de Estudios del Padre de la A Serna". En Hernández de Alba Guillermo. Documentos para la Historia de la Educación en Colombia. Volumen VII, Tunja. RUDECOLOMBIA. 1985

Restrepo Posada, José. "Rectores del Colegio Seminario de San Bartolomé" En Revista Javeriana No 187. Vol 38 (1952) 
Torres, Camilo. “El doctor Camilo Torres, catedrático del Colegio Mayor de Nuestra Señora del Rosario, por encargo del claustro de doctores de la Universidad Tomística, analiza el plan de estudios para el Colegio de Medellín. Va firmado por el rector y claustro de la Universidad Hernández de Alba Documentos para la Historia de la Educación en Colombia Tunja: RUDECOLOMBIA

Uribe Ángel, Jorge Tomás. “La Universidad tardocolonial latinoamericana: El caso de Santafé en La Nueva Granada. 1800-1810". Revista Historia de la Educación Latinoamericana. Vol. 16 No, 22, (2014): 139 $-156$. 